\title{
An Energy Gap for Complex Yang-Mills Equations
}

Teng HUANG ${ }^{\dagger \dagger}$

$\dagger$ Key Laboratory of Wu Wen-Tsun Mathematics, Chinese Academy of Sciences, P.R. China

$\ddagger$ School of Mathematical Sciences, University of Science and Technology of China, P.R. China

E-mail: oula143@mail.ustc.edu.cn

Received May 31, 2017, in final form July 26, 2017; Published online August 08, 2017

https://doi.org/10.3842/SIGMA.2017.061

\begin{abstract}
We use the energy gap result of pure Yang-Mills equation [Feehan P.M.N., Adv. Math. 312 (2017), 547-587] to prove another energy gap result of complex Yang-Mills equations [Gagliardo M., Uhlenbeck K., J. Fixed Point Theory Appl. 11 (2012), 185-198], when Riemannian manifold $X$ of dimension $n \geq 2$ satisfies certain conditions.
\end{abstract}

Key words: complex Yang-Mills equations; energy gap; gauge theory

2010 Mathematics Subject Classification: 58E15; 81T13

\section{Introduction}

Let $X$ be an oriented $n$-manifold endowed with a smooth Riemannian metric $g$. Let $P$ be a principle $G$-bundle over $X$. The structure group $G$ is assumed to be a compact Lie group with Lie algebra $\mathfrak{g}$. We denote a connection on $P$ as $A$ and its curvature as $F_{A}$. Let $\mathfrak{g}_{P}$ be the adjoint bundle of $P$. We define by $\mathrm{d}_{A}$ the exterior covariant derivative on section of $\Lambda^{\bullet} T^{*} X \otimes\left(P \times_{G} \mathfrak{g}_{P}\right)$ with respect to the connection $A$. The curvature $\mathcal{F}_{\mathcal{A}}$ of the complex connection $\mathcal{A}:=\mathrm{d}_{A}+\sqrt{-1} \phi$, $\phi \in \Omega^{1}\left(X, \mathfrak{g}_{P}\right)$ is a two-form with values in $P \times_{G}\left(\mathfrak{g}_{P}^{\mathbb{C}}\right)$ :

$$
\mathcal{F}_{\mathcal{A}}=\left[\left(\mathrm{d}_{A}+\sqrt{-1} \phi\right) \wedge\left(\mathrm{d}_{A}+\sqrt{-1} \phi\right)\right]=F_{A}-\frac{1}{2}[\phi \wedge \phi]+\sqrt{-1} \mathrm{~d}_{A} \phi .
$$

The complex Yang-Mills functional is defined in any dimension as the norm squared of the complex curvature [9, Section 3]

$$
\mathrm{YM}_{\mathbb{C}}(A, \phi):=\int_{X}\left|\mathcal{F}_{\mathcal{A}}\right|^{2}=\int_{X}\left(\left|F_{A}-\phi \wedge \phi\right|^{2}+\left|\mathrm{d}_{A} \phi\right|^{2}\right) .
$$

This functional reduces to the pure Yang-Mills functional when the extra field $\phi$ vanishes. The Euler-Lagrange equations for this functional are

$$
\begin{aligned}
& \mathrm{d}_{A}^{*}\left(F_{A}-\phi \wedge \phi\right)+(-1)^{n} *\left[\phi, * \mathrm{~d}_{A} \phi\right]=0, \\
& \mathrm{~d}_{A}^{*} \mathrm{~d}_{A} \phi-(-1)^{n} *\left[\phi, *\left(F_{A}-\phi \wedge \phi\right)\right]=0 .
\end{aligned}
$$

They can also be succinctly written as

$$
\mathrm{d}_{\mathcal{A}}^{*} \mathcal{F}_{\mathcal{A}}=0
$$

These equations are not elliptic, even after the real gauge-equivalence, so it is necessary to add the moment map condition

$$
\mathrm{d}_{A}^{*} \phi=0 .
$$

In this article, we call $\mathrm{d}_{A}+\sqrt{-1} \phi$ the solution of the complex Yang-Mills equations, it not only satisfies equations (1.1), but also satisfies the moment map condition. These equations called complex just because the connection $\mathrm{d}_{A}+\sqrt{-1} \phi$ is a 1 -form with value in $P \times_{G}\left(\mathfrak{g}_{\mathbb{C}}\right)$, the base manifold is always a real Riemannian manifold. 
Remark 1.1. Some examples of complex Yang-Mills equations:

(1) Kapustin-Witten equations [9]

$$
\left(F_{A}-\phi \wedge \phi\right)^{+}=0, \quad\left(\mathrm{~d}_{A} \phi\right)^{-}=0, \quad \mathrm{~d}_{A} * \phi=0
$$

on four-dimensional manifolds.

(2) On a stable Higgs bundle $(E, \theta)$, there exists a Hermitian metric $h$ such that the HitchinSimpson connection $\mathrm{d}_{A_{h}}+\theta+\theta^{*, h}$ satisfies the Einstein condition [12, 16]

$$
\sqrt{-1} \Lambda_{\omega}\left(F_{A_{h}}+\left[\theta, \theta^{*, h}\right]\right)-\lambda \operatorname{Id}_{E}=0,
$$

the connection $\mathrm{d}_{A_{h}}+\theta+\theta^{*, h}$ also satisfies complex Yang-Mills equations.

In particular, it is easy to see that if $\phi=0$, the complex Yang-Mills equations will reduce to the pure Yang-Mills equation $\mathrm{d}_{A}^{*} F_{A}=0$. Many researchers have studied the energy gap of Yang-Mills equation. The motivation of these gap results is partly from physics and partly from math which would be help to better understand the behavior of the Yang-Mills functional near its critical points. In $[1,2,3,10,14]$, they all require some positive hypotheses on the curvature tensors of a Riemannian metric. In [8], Feehan applied the Lojasiewicz-Simon gradient inequality [8, Theorem 3.2] to remove the positive hypothesis on the Riemannian curvature tensors. In [13], the author gave another proof of energy gap theorem of pure Yang-Mills equation without using Lojasiewicz-Simon gradient inequality. We also want to understand the behaviour of the complex Yang-Mills functional near its critical points. It is an interesting question to consider, whether the complex Yang-Mills equations have the energy gap phenomenon. In this article, we give a positive answer to this question when $X$ satisfies certain conditions.

Theorem 1.2. Let $X$ be a closed, oriented, smooth Riemannian manifold of dimension $n \geq 2$ with smooth Riemannan metric $g, P$ be a $G$-bundle over $X$ with $G$ being a compact Lie group, let $2 p>n$ when $n \neq 2,4$ or $p \geq 2$ when $n=2,4$. Then there is a positive constant $\varepsilon=\varepsilon(n, p, g)$ with the following significance. Suppose that all flat connections on $P$ are non-degenerate in the sense of Definition 3.5. If the pair $(A, \phi)$ is a $C^{\infty}$-solution of complex Yang-Mills equations over $X$, the curvature $F_{A}$ of connection $A$ obeys

$$
\left\|F_{A}\right\|_{L^{p}(X)} \leq \varepsilon
$$

then $A$ is a flat connection and $\phi$ must vanish.

Remark 1.3. In fact, the extra fields will vanish if the Ricci tensor of a Riemannian metric of $X$ is positive (see Corollary 2.3). For a general Riemannian metric, we will not know whether $\left.\operatorname{ker} \Delta_{\Gamma}\right|_{\Omega^{1}\left(X, \mathfrak{g}_{P}\right)}=\{0\}$ ( $\Gamma$ is any flat connection on $P$ ) unless we assume some topological hypotheses for $X$, such as $\pi_{1}(X)=\{1\}$, so $P \cong X \times G$ if and only if $P$ is flat [5, Theorem 2.2.1]. In this case, $\Gamma$ is gauge-equivalent to the product connection and $\left.\operatorname{ker} \Delta_{\Gamma}\right|_{\Omega^{1}\left(X, \mathfrak{g}_{P}\right)} \cong H^{1}(X, \mathbb{R})$, so the hypothesis for $X$ ensures that the kernel vanishes.

If $\mathcal{F}_{\mathcal{A}}=0$, i.e., $F_{A}-\phi \wedge \phi=0$ and $\mathrm{d}_{A} \phi=0$, then we call $\mathcal{A}=\mathrm{d}_{A}+\sqrt{-1} \phi$ a complex flat connection. Now, we denote the moduli space of solutions of complex flat-connections by

$$
\mathcal{M}(P, g):=\left\{(A, \phi) \mid\left(F_{A}-\phi \wedge \phi\right)=0 \text { and } \mathrm{d}_{A} \phi=\mathrm{d}_{A}^{*} \phi=0\right\} / \mathcal{G}_{P} .
$$

In particular, the moduli space $M(P, g)$ can be embedded into $\mathcal{M}(P, g)$ via $A \mapsto(A, 0)$, where

$$
M(P, g):=\left\{\Gamma: F_{\Gamma}=0\right\} / \mathcal{G}_{P},
$$

is the moduli space of gauge-equivalence class $[\Gamma]$ of flat connection $\Gamma$ on $P$. Obviously, the complex flat connection also satisfies the complex Yang-Mills equations. Using the gap Theorem 1.2 of complex Yang-Mills equations, we can have a gap result for the extra fields as follows 
Corollary 1.4. Let $X$ be a closed, oriented, smooth Riemannian manifold of dimension $n \geq 2$ with smooth Riemannan metric $g, P$ be a $G$-bundle over $X$ with $G$ being a compact Lie group. Then there is a positive constant $\varepsilon=\varepsilon(g, n)$ with the following significance. Suppose that all flat connections on $P$ are non-degenerate in the sense of Definition 3.5. If the pair $(A, \phi)$ is a $C^{\infty}$-solution of complex flat connection over $X$, the $L^{2}$-norm of extra field $\phi$ obeys

$$
\|\phi\|_{L^{2}(X)} \leq \varepsilon
$$

then $\phi$ vanishes and $A$ is a flat connection. In particular, if $M(P, g)$ and $\mathcal{M}(P, g) \backslash M(P, g)$ are both not empty, then the moduli space $\mathcal{M}(P, g)$ is non-connected.

The organization of this paper is as follows. In Section 2, first we set our notations and recall some basic definitions in differential geometry. Next, we recall some identities and some estimates for the solutions of complex Yang-Mills equations which were proved by Gagliardo and Uhlenbeck [9]. Finally, we recall an energy gap result of the pure Yang-Mills equation due to Feehan [8]. Since the Theorem 2.6 plays an essential role in our proof of our main result, we provide more details to prove the theorem by another method. In Section 3, we define the least eigenvalue $\lambda(A)$ of $\mathrm{d}_{A} \mathrm{~d}_{A}^{*}+\left.\mathrm{d}_{A}^{*} \mathrm{~d}_{A}\right|_{\Omega^{1}\left(X, \mathfrak{g}_{P}\right)}$ with respect to connection $A$. We extend the idea of Feehan [6] to prove that $\lambda(A)$ has a lower bound that is uniform with respect to $[A]$ obeying $\left\|F_{A}\right\|_{L^{p}(X)} \leq \varepsilon$ for a small enough $\varepsilon=\varepsilon(g, n, p)$ under some conditions for $g, G, P$, and $X$. We conclude Section 4 with the proofs of Theorem 1.2 and Corollary 1.4.

\section{Fundamental preliminaries}

We shall generally adhere to the now standard gauge-theory conventions and notation of Donaldson and Kronheimer [5] and Feehan [8]. Throughout our article, $G$ denotes a compact Lie group and $P$ a smooth principal $G$-bundle over a compact Riemannnian manifold $X$ of dimension $n \geq 2$ and endowed with Riemannian metric $g, \mathfrak{g}_{P}$ denote the adjoint bundle of $P$, endowed with a $G$-invariant inner product and $\Omega^{p}\left(X, \mathfrak{g}_{P}\right)$ denote the smooth $p$-forms with values in $\mathfrak{g}_{P}$. Given a connection on $P$, we denote by $\nabla_{A}$ the corresponding covariant derivative on $\Omega^{*}\left(X, \mathfrak{g}_{P}\right)$ induced by $A$ and the Levi-Civita connection of $X$. Let $\mathrm{d}_{A}$ denote the exterior derivative associated to $\nabla_{A}$.

For $u \in L^{p}\left(X, \mathfrak{g}_{P}\right)$, where $1 \leq p<\infty$ and $k$ is an integer, we denote

$$
\|u\|_{L_{k, A}^{p}(X)}:=\left(\sum_{j=0}^{k} \int_{X}\left|\nabla_{A}^{j} u\right|^{p} \operatorname{dvol}_{g}\right)^{1 / p},
$$

where $\nabla_{A}^{j}:=\nabla_{A} \circ \cdots \circ \nabla_{A}$ (repeated $j$ times for $j \geq 0$ ). For $p=\infty$, we denote

$$
\|u\|_{L_{k, A}^{\infty}(X)}:=\sum_{j=0}^{k} \operatorname{ess} \sup _{X}\left|\nabla_{A}^{j} u\right| .
$$

\subsection{Identities for the solutions}

In this section, we recall some basic identities that the solutions to complex Yang-Mills connections obey. A nice discussion of these identities can be found in [9].

Theorem 2.1 (Weitezenböck formula).

$$
\mathrm{d}_{A}^{*} \mathrm{~d}_{A}+\mathrm{d}_{A} \mathrm{~d}_{A}^{*}=\nabla_{A}^{*} \nabla_{A}+\operatorname{Ric}(\cdot)+*\left[* F_{A}, \cdot\right] \quad \text { on } \quad \Omega^{1}\left(X, \mathfrak{g}_{P}\right),
$$

where Ric is the Ricci tensor. 
Proposition 2.2 ([9, Theorem 4.3]). If $\mathrm{d}_{A}+\sqrt{-1} \phi$ is a solution of the complex Yang-Mills equations, then

$$
\nabla_{A}^{*} \nabla_{A} \phi+\operatorname{Ric} \circ \phi+*[*(\phi \wedge \phi), \phi]=0 .
$$

By integrating (2.2) over $X$, we have an identity

$$
\left\|\nabla_{A} \phi\right\|_{L^{2}(X)}^{2}+\langle\operatorname{Ric} \circ \phi, \phi\rangle_{L^{2}(X)}+2\|\phi \wedge \phi\|_{L^{2}(X)}^{2}=0 .
$$

Then the results by Gagliardo-Uhlenbeck give a following consequence result for the extra fields.

Corollary 2.3 ([9, Corollary 4.5]). If $X$ is a compact manifold with a positive Ricci curvature, then solutions of the complex Yang-Mills equations reduce to solutions of the pure Yang-Mills equation with $\phi=0$.

Proposition 2.4 (energy identity). If $\mathrm{d}_{A}+\sqrt{-1} \phi$ is a solution of the complex Yang-Mills equations, then

$$
\mathrm{YM}_{\mathbb{C}}(A, \phi)=\left\|F_{A}\right\|_{L^{2}(X)}^{2}-\|\phi \wedge \phi\|_{L^{2}(X)}^{2} .
$$

Proof. By using the moment condition $\mathrm{d}_{A}^{*} \phi=0$, the complex Yang-Mills functional is written as

$$
\begin{aligned}
\mathrm{YM}_{\mathbb{C}}(A, \phi) & =\int_{X}\left(\left|F_{A}-\phi \wedge \phi\right|^{2}+\left|\mathrm{d}_{A} \phi\right|^{2}+\left|\mathrm{d}_{A}^{*} \phi\right|\right) \\
& =\int_{X}\left|F_{A}\right|^{2}+|\phi \wedge \phi|^{2}-2\left\langle F_{A}, \phi \wedge \phi\right\rangle+\left|\mathrm{d}_{A} \phi\right|^{2}+\left|\mathrm{d}_{A}^{*} \phi\right| \\
& =\int_{X}\left|F_{A}\right|^{2}+|\phi \wedge \phi|^{2}+\left|\nabla_{A} \phi\right|^{2}+\langle\operatorname{Ric} \circ \phi, \phi\rangle \\
& =\left\|F_{A}\right\|_{L^{2}(X)}^{2}-\|\phi \wedge \phi\|_{L^{2}(X)}^{2} .
\end{aligned}
$$

For the last identity, we use the equation (2.3).

As an application of the maximum principle, Gagliardo-Uhlenbeck obtain a priori $L^{\infty}$ estimate for the extra fields.

Theorem 2.5 ([9, Corollary 4.6]). Let $G$ be a compact Lie group, $P$ be a G-bundle over a closed, smooth manifold $X$ of dimension $n \geq 2$ and endowed with a smooth Riemannian metric $g$. Then there is a positive constant $C=C(g, n)$ with the following significance. If $(A, \phi)$ is a smooth solution of complex Yang-Mills equation, then

$$
\|\phi\|_{L^{\infty}(X)} \leq C\|\phi\|_{L^{2}(X)} .
$$

\subsection{Energy gap for Yang-Mills connections}

In this section, first we recall an energy gap result of Yang-Mills equation.

Theorem 2.6 ([8, Theorem 1.1]). Let $X$ be a closed, oriented, smooth Riemannian manifold of dimension $n \geq 2$ with smooth Riemannan metric $g, P$ be a G-bundle over $X$, let $2 p \geq n$ when $n \geq 3$ or $p \geq 2$ when $n=2$. Then any Yang-Mills connection $A$ over $X$ with compact Lie group $G$ is either satisfies

$$
\int_{X}\left|F_{A}\right|^{p} \mathrm{dvol}_{g} \geq C_{0}
$$

for a constant $C_{0}>0$ depending only on $X, n, p, G$ or the connection $A$ is flat. 
In [13], the author proves the energy gap theorem of Yang-Mills connection without using the Lojasiewicz-Simon gradient inequality. Here, we give a proof in detail for the readers convenience. We review a key result due to Uhlenbeck for the connections with $L^{p}$-small curvature $(2 p>n)$ [20] which provides existence of a flat connection $\Gamma$ on $P$, a global gauge transformation $u$ of $A$ to Coulomb gauge with respect to $\Gamma$ and a Sobolev norm estimate for the distance between $\Gamma$ and $A$.

Theorem 2.7 ([20, Corollary 4.3] and [8, Theorem 5.1]). Let $X$ be a closed, smooth manifold of dimension $n \geq 2$ and endowed with a Riemannian metric $g$, and $G$ be a compact Lie group, and $2 p>n$. Then there are constants, $\varepsilon=\varepsilon(n, g, G, p) \in(0,1]$ and $C=C(n, g, G, p) \in[1, \infty)$, with the following significance. Let $A$ be a $L_{1}^{p}$ connection on a principal $G$-bundle $P$ over $X$. If the curvature $F_{A}$ obeys

$$
\left\|F_{A}\right\|_{L^{p}(X)} \leq \varepsilon
$$

then there exists a flat connection, $\Gamma$, on $P$ and a gauge transformation $u \in L_{2}^{p}(X)$ such that

(1) $\mathrm{d}_{\Gamma}^{*}\left(u^{*}(A)-\Gamma\right)=0$ on $X$,

(2) $\left\|u^{*}(A)-\Gamma\right\|_{L_{1, \Gamma}^{p}} \leq C\left\|F_{A}\right\|_{L^{p}(X)}$, and

(3) $\left\|u^{*}(A)-\Gamma\right\|_{L_{1, \Gamma}^{\frac{n}{2}}} \leq C\left\|F_{A}\right\|_{L^{\frac{n}{2}}(X)}$.

Next, we also review another key result due to Uhlenbeck [18, Theorem 3.5] concerning a priori estimate for the curvature of a Yang-Mills connection over a closed Riemannian manifold.

Theorem 2.8 ([8, Corollary 4.6]). Let $X$ be a compact manifold of dimension $n \geq 3$ and endowed with a Riemannian metric $g$, let $A$ be a smooth Yang-Mills connection with respect to the metric $g$ on a smooth $G$-bundle $P$ over $X$. Then there exist constants $\varepsilon=\varepsilon(n, g)>0$ and $C=C(n, g)$ with the following significance. If the curvature $F_{A}$ obeys

$$
\left\|F_{A}\right\|_{L^{\frac{n}{2}}(X)} \leq \varepsilon,
$$

then

$$
\left\|F_{A}\right\|_{L^{\infty}(X)} \leq C\left\|F_{A}\right\|_{L^{2}(X)} .
$$

Proof Theorem 2.6. For any $p \geq 2 n(n \geq 3)$, the estimate in Theorem 2.8 yields

$$
\left\|F_{A}\right\|_{L^{p}(X)} \leq C\left\|F_{A}\right\|_{L^{\infty}(X)} \leq C\left\|F_{A}\right\|_{L^{2}(X)},
$$

for $C=C(g, n)$. If $n \geq 4$, then

$$
\left\|F_{A}\right\|_{L^{2}(X)} \leq C\left\|F_{A}\right\|_{L^{\frac{n}{2}}(X)} .
$$

If $n=3$, the $L^{p}$ interpolation [11, equation (7.9)] implies that

$$
\left\|F_{A}\right\|_{L^{2}(X)} \leq C\left\|F_{A}\right\|_{L^{\frac{3}{2}(X)}}^{3 / 4}\left\|F_{A}\right\|_{L^{\infty}(X)}^{1 / 4} \leq C\left\|F_{A}\right\|_{L^{\frac{3}{2}}(X)}^{3 / 4}\left\|F_{A}\right\|_{L^{2}(X)}^{1 / 4},
$$

and thus

$$
\left\|F_{A}\right\|_{L^{2}(X)} \leq C\left\|F_{A}\right\|_{L^{\frac{3}{2}}(X)} .
$$

Therefore, combining (2.4)-(2.6), we obtain

$$
\left\|F_{A}\right\|_{L^{p}(X)} \leq C\left\|F_{A}\right\|_{L^{n / 2}(X)}, \quad \forall 2 p \geq n \quad \text { and } \quad n \geq 3 .
$$


Hence, if we suppose $\left\|F_{A}\right\|_{L^{s}(X)}$ sufficiently small ( $2 s \geq n$ when $n \geq 3$ or $s \geq 2$ when $\left.n=2\right)$ such that $\left\|F_{A}\right\|_{L^{q}(X)}(2 q>n$ when $n \geq 3$ or $q \geq 2$ when $n=2)$ satisfies the hypothesis of Theorem 2.7, then Theorem 2.7 will provide a flat connection $\Gamma$ on $P$, and a gauge transformation $u \in \mathcal{G}_{P}$ and the estimate

$$
\left\|u^{*}(A)-\Gamma\right\|_{L_{1}^{q}(X)} \leq C(q)\left\|F_{A}\right\|_{L^{q}(X)},
$$

and

$$
\mathrm{d}_{\Gamma}^{*}\left(u^{*}(A)-\Gamma\right)=0 .
$$

We denote $\tilde{A}:=u^{*}(A)$ and $a:=u^{*}(A)-\Gamma$, then the curvature of $\tilde{A}$ is

$$
F_{\tilde{A}}=\mathrm{d}_{\Gamma} a+a \wedge a .
$$

The connection $\tilde{A}$ also satisfies Yang-Mills equation

$$
0=\mathrm{d}_{\tilde{A}}^{*} F_{\tilde{A}} .
$$

Hence taking the $L^{2}$-inner product of (2.7) with $a$, we obtain

$$
\begin{aligned}
0 & =\left(\mathrm{d}_{\tilde{A}}^{*} F_{\tilde{A}}, a\right)_{L^{2}(X)}=\left(F_{\tilde{A}}, \mathrm{~d}_{\tilde{A}} a\right)_{L^{2}(X)} \\
& =\left(F_{\tilde{A}}, \mathrm{~d}_{\Gamma} a+2 a \wedge a\right)_{L^{2}(X)}=\left(F_{\tilde{A}}, F_{\tilde{A}}+a \wedge a\right)_{L^{2}(X)} .
\end{aligned}
$$

Then we get

$$
\begin{aligned}
\left\|F_{A}\right\|_{L^{2}(X)}^{2} & =\left\|F_{\tilde{A}}\right\|_{L^{2}(X)}^{2}=-\left(F_{\tilde{A}}, a \wedge a\right)_{L^{2}(X)} \\
& \leq\left\|F_{\tilde{A}}\right\|_{L^{2}(X)}\|a \wedge a\|_{L^{2}(X)}=\left\|F_{A}\right\|_{L^{2}(X)}\|a \wedge a\|_{L^{2}(X)},
\end{aligned}
$$

here we use the fact $\left|F_{u^{*}(A)}\right|=\left|F_{A}\right|$ since $F_{u^{*}(A)}=u \circ F_{A} \circ u^{-1}$.

If $n \geq 4$,

$$
\begin{aligned}
\|a \wedge a\|_{L^{2}(X)} & \leq C\|a\|_{L^{4}(X)}^{2} \leq C\|a\|_{L^{n}(X)}^{2} \leq C\|a\|_{L_{1}^{\frac{n}{2}}(X)}^{2} \\
& \leq C\left\|F_{A}\right\|_{L^{\frac{n}{2}(X)}}^{2} \leq C\left\|F_{A}\right\|_{L^{\infty}(X)}^{2} \leq C\left\|F_{A}\right\|_{L^{2}(X)}^{2},
\end{aligned}
$$

here we apply the Sobolev embedding $L_{1}^{\frac{n}{2}} \hookrightarrow L^{n}$.

If $n=2,3$,

$$
\|a \wedge a\|_{L^{2}(X)} \leq C\|a\|_{L^{4}(X)}^{2} \leq C\|a\|_{L_{1}^{2}(X)}^{2} \leq C\left\|F_{A}\right\|_{L^{2}(X)}^{2},
$$

here we apply the Sobolev embedding $L_{1}^{2} \hookrightarrow L^{4}$.

Combining the preceding inequalities we have

$$
\left\|F_{A}\right\|_{L^{2}(X)}^{2} \leq C\left\|F_{A}\right\|_{L^{2}(X)}^{3} .
$$

We can choose $\left\|F_{A}\right\|_{L^{2}(X)}$ sufficiently small such that $C\left\|F_{A}\right\|_{L^{2}(X)}<1$, hence $\left\|F_{A}\right\|_{L^{2}(X)} \equiv 0$ and thus $A$ must be a flat connection. Then we complete the proof.

\section{Eigenvalue bounds for Laplacian $\Delta_{A}$}

In this section, we will show that the least eigenvalue $\lambda(\Gamma)$ of $\mathrm{d}_{\Gamma}^{*} \mathrm{~d}_{\Gamma}+\mathrm{d}_{\Gamma} \mathrm{d}_{\Gamma}^{*}$ has a positive lower bound $\lambda$ that is uniform with respect to $[\Gamma] \in M(P, g)$ under the given conditions on $X$ and $P$. The method is similar to Feehan [7] that has proved the least eigenvalue $\mu_{g}(A)$ of $\mathrm{d}_{A}^{+, g} \mathrm{~d}_{A}^{+, * g}$ which has a positive lower bound $\mu_{0}$ that is uniform with respect to $[A] \in \mathcal{B}(P, g)$ obeys $\left\|F_{A}^{+, g}\right\|_{L^{2}} \leq \varepsilon$, for a small enough $\varepsilon$ and under the given sets of conditions on $g, G, P$ and $X$. 


\subsection{Continuity for the least eigenvalue of $\Delta_{A}$}

From Uhlenbeck compactness theorem [19, 21], we know

Proposition 3.1. Let $G$ be a compact Lie group, $P$ be a $G$-bundle over a closed, smooth manifold $X$ of dimension $n \geq 2$ and endowed with a smooth Riemannian metric $g$. Then the moduli space $M(P, g)$ is compact.

The definition of the least eigenvalue of $\Delta_{A}$ on $L^{2}\left(X, \Omega^{1}\left(\mathfrak{g}_{P}\right)\right)$ is similar to [17, Definition 3.1].

Definition 3.2 (least eigenvalue of $\Delta_{A}$ ). Let $G$ be a compact Lie group, $P$ be a $G$-bundle over a closed, smooth manifold $X$ of dimension $n \geq 2$ and endowed with a smooth Riemannian metric $g$. Let $A$ be a connection of Sobolev class $L_{1}^{2}$ on $P$. The least eigenvalue of $\Delta_{A}$ on $L^{2}\left(X, \Omega^{1}\left(\mathfrak{g}_{P}\right)\right)$ is

$$
\lambda(A):=\inf _{v \in \Omega^{1}\left(\mathfrak{g}_{P}\right) \backslash\{0\}} \frac{\left\langle\Delta_{A} v, v\right\rangle_{L^{2}}}{\|v\|^{2}} .
$$

The method to prove the continuity of the least eigenvalue of $\Delta_{A}$ with respect to the connection is similar to one by means of which Feehan proved continuity of the least eigenvalue of $\mathrm{d}_{A}^{+} \mathrm{d}_{A}^{+, *}$ with respect to the connection in $[6,7]$.

We give a priori estimate for $v \in \Omega^{1}\left(X, \mathfrak{g}_{P}\right)$ when the curvature $F_{A}$ is sufficiently small in some $L^{p}$-norms.

Lemma 3.3. Let $G$ be a compact Lie group, $P$ be a G-bundle over a closed, smooth manifold $X$ of dimension $n \geq 2$ and endowed with a smooth Riemannian metric $g$, let $2 p \geq n$ when $n \geq 3$ or $p>1$ when $n=2$. Then there are positive constants, $c=c(g, p)$ and $\varepsilon=\varepsilon(g, p) \in(0,1]$, with the following significance. If $A$ is a connection on $P$ over $X$ such that

$$
\left\|F_{A}\right\|_{L^{p}(X)} \leq \varepsilon
$$

and $v \in \Omega^{1}\left(X, \mathfrak{g}_{P}\right)$, then

$$
\|v\|_{L_{1}^{2}(X)}^{2} \leq c\left(\left\|\mathrm{~d}_{A} v\right\|_{L^{2}(X)}^{2}+\left\|\mathrm{d}_{A}^{*} v\right\|_{L^{2}(X)}^{2}+\|v\|_{L^{2}(X)}^{2}\right) .
$$

Proof. The Weitzenböck formula for $v \in \Omega^{1}\left(X, \mathfrak{g}_{P}\right)$, namely,

$$
\left(\mathrm{d}_{A} \mathrm{~d}_{A}^{*}+\mathrm{d}_{A}^{*} \mathrm{~d}_{A}\right) v=\nabla_{A}^{*} \nabla_{A} v+\operatorname{Ric} \circ v+*\left[* F_{A}, v\right] .
$$

Hence

$$
\left\|\nabla_{A} v\right\|_{L^{2}(X)}^{2} \leq\left\|\mathrm{d}_{A}^{*} v\right\|_{L^{2}(X)}^{2}+\left\|\mathrm{d}_{A} v\right\|_{L^{2}(X)}^{2}+c\|v\|_{L^{2}(X)}^{2}+\left|\left\langle *\left[* F_{A}, v\right], v\right\rangle_{L^{2}(X)}\right|,
$$

where $c=c(g)$.

If $n \geq 3$, by Hölder inequality, we see that

$$
\begin{aligned}
\left|\left\langle *\left[* F_{A}, v\right], v\right\rangle_{L^{2}(X)}\right| & \leq\left\|F_{A}\right\|_{L^{n / 2}(X)}\|v\|_{L^{2 n /(n-2)}(X)}^{2} \\
& \leq c\left\|F_{A}\right\|_{L^{n / 2}(X)}\|v\|_{L_{1}^{2}(X)}^{2} \leq c\left\|F_{A}\right\|_{L^{p}(X)}\|v\|_{L_{1}^{2}(X)}^{2},
\end{aligned}
$$

for some $c=c(g)$.

If $n=2$, define $q \in(1, \infty)$ by $1 / q=1-1 / p$, we have

$$
\left|\left\langle *\left[* F_{A}, v\right], v\right\rangle_{L^{2}(X)}\right| \leq\left\|F_{A}\right\|_{L^{p}(X)}\|v\|_{L^{2 q}(X)}^{2} \leq c\left\|F_{A}\right\|_{L^{p}(X)}\|v\|_{L_{1}^{2}(X)}^{2},
$$

here we use the Sobolev embedding $L_{1}^{2} \hookrightarrow L^{2 q}$. 
Combining of the preceding inequalities and Kato inequality $|\nabla| v|| \leq\left|\nabla_{A} v\right|$ yields

$$
\begin{aligned}
\|v\|_{L_{1}^{2}(X)}^{2} & \leq\left(\left\|\nabla_{A} v\right\|_{L^{2}(X)}^{2}+\|v\|_{L^{2}(X)}^{2}\right) \\
& \leq\left\|\mathrm{d}_{A}^{*} v\right\|_{L^{2}(X)}^{2}+\left\|\mathrm{d}_{A} v\right\|_{L^{2}(X)}^{2}+(c+1)\|v\|_{L^{2}(X)}+c\left\|F_{A}\right\|_{L^{p}(X)}\|v\|_{L_{1}^{2}(X)}^{2},
\end{aligned}
$$

for some $c=c(g)$. Provided $c\left\|F_{A}\right\|_{L^{p}(X)} \leq 1 / 2$, rearrangements gives (3.3).

Following the idea of [6, Lemma 35.12], we also have a useful lemma.

Lemma 3.4 ( $L^{2 p}$-continuity of least eigenvalue of $\Delta_{A}$ with respect to the connection). Let $G$ be a compact Lie group, $P$ be a $G$-bundle over a closed, smooth manifold $X$ of dimension $n \geq 2$ and endowed with a smooth Riemannian metric $g$, let $2 p \geq n$ or $n \geq 3$ and $p>1$ when $n=2$. Then there are positive constants, $C=C(g, p)$ and $\varepsilon=\varepsilon(g, p)$, with the following significance. If $A_{0}, A$ are smooth connections on $P$ that obey the curvatures bounded (3.2) and

$$
\left\|A-A_{0}\right\|_{L^{2 p}(X)} \leq \varepsilon
$$

then, we denote $a:=A-A_{0}$,

$$
\left(1-C\|a\|_{L^{2 p}(X)}^{2}\right) \lambda\left(A_{0}\right)-C\|a\|_{L^{2 p}(X)}^{2} \leq \lambda(A) \leq\left(1-C\|a\|_{L^{2 p}(X)}^{2}\right)^{-1}\left(\lambda\left(A_{0}\right)+C\|a\|_{L^{2 p}(X)}^{2}\right) .
$$

Proof. For convenience, write $a:=A-A_{0} \in L^{n}\left(X, \Omega^{1} \otimes \mathfrak{g}_{P}\right)$. For $v \in L_{1}^{2}\left(X, \Omega^{1} \otimes \mathfrak{g}_{P}\right)$, we have $\mathrm{d}_{A} v=\mathrm{d}_{A_{0}} v+[a, v]$.

If $n \geq 3$, by Hölder inequality

$$
\begin{aligned}
\left\|\mathrm{d}_{A} v\right\|_{L^{2}(X)}^{2} & =\left\|\mathrm{d}_{A_{0}} v+[a, v]\right\|_{L^{2}(X)}^{2} \geq\left\|\mathrm{d}_{A_{0}} v\right\|_{L^{2}(X)}^{2}-\|[a, v]\|_{L^{2}(X)}^{2} \\
& \geq\left\|\mathrm{d}_{A_{0}} v\right\|_{L^{2}(X)}^{2}-2\|a\|_{L^{n}(X)}^{2}\|v\|_{L^{2 n /(n-2)}(X)}^{2} \\
& \geq\left\|\mathrm{d}_{A_{0}} v\right\|_{L^{2}(X)}^{2}-2 c_{1}\|a\|_{L^{n}(X)}^{2}\|v\|_{L_{1, A_{0}}^{2}(X)}^{2} \\
& \geq\left\|\mathrm{d}_{A_{0}} v\right\|_{L^{2}(X)}^{2}-2 c_{1}\|a\|_{L^{2 p}(X)}^{2}\|v\|_{L_{1, A_{0}}^{2}(X)}^{2},
\end{aligned}
$$

where $c_{1}=c_{1}(g)$ is the Sobolev embedding constant for $L_{1}^{2} \hookrightarrow L^{2 n /(n-2)}$.

Similarly, $\mathrm{d}_{A}^{*} v=\mathrm{d}_{A_{0}}^{*} v \pm *[a, * v]$ and

$$
\begin{aligned}
\left\|\mathrm{d}_{A}^{*} v\right\|_{L^{2}(X)}^{2} & =\left\|\mathrm{d}_{A_{0}}^{*} v \pm *[a, * v]\right\|_{L^{2}(X)}^{2} \geq\left\|\mathrm{d}_{A_{0}}^{*} v\right\|_{L^{2}(X)}^{2}-\|[a, * v]\|_{L^{2}(X)}^{2} \\
& \geq\left\|\mathrm{d}_{A_{0}}^{*} v\right\|_{L^{2}(X)}^{2}-2\|a\|_{L^{n}(X)}^{2}\|v\|_{L^{2 n /(n-2)}(X)}^{2} \\
& \geq\left\|\mathrm{d}_{A_{0}}^{*} v\right\|_{L^{2}(X)}^{2}-2 c_{1}\|a\|_{L^{n}(X)}^{2}\|v\|_{L_{1, A_{0}}^{2}(X)}^{2} \\
& \geq\left\|\mathrm{d}_{A_{0}} v\right\|_{L^{2}(X)}^{2}-2 c_{1}\|a\|_{L^{2 p}(X)}^{2}\|v\|_{L_{1, A_{0}}^{2}(X)}^{2} .
\end{aligned}
$$

If $n=2$, define $q \in(1, \infty)$ by $1=1 / p+1 / q$,

$$
\begin{aligned}
\left\|\mathrm{d}_{A} v\right\|_{L^{2}(X)}^{2} & =\left\|\mathrm{d}_{A_{0}} v+[a, v]\right\|_{L^{2}(X)}^{2} \geq\left\|\mathrm{d}_{A_{0}} v\right\|_{L^{2}(X)}^{2}-\|[a, v]\|_{L^{2}(X)}^{2} \\
& \geq\left\|\mathrm{d}_{A_{0}} v\right\|_{L^{2}(X)}^{2}-2\|a\|_{L^{2 p}(X)}^{2}\|v\|_{L^{2 q}(X)}^{2} \\
& \geq\left\|\mathrm{d}_{A_{0}} v\right\|_{L^{2}(X)}^{2}-2 c_{1}\|a\|_{L^{2 p}(X)}^{2}\|v\|_{L_{1, A_{0}}^{2}(X)}^{2},
\end{aligned}
$$

here we use the Sobolev embedding $L_{1}^{2} \hookrightarrow L^{2 q}$.

Applying the a priori estimate (3.3) for $\|v\|_{L_{1}^{2}(X)}$ from Lemma 3.3, with $c=c(g)$ and smooth enough $\varepsilon=\varepsilon(g)$, we get

$$
\|v\|_{L_{1}^{2}(X)}^{2} \leq c\left(\left\|\mathrm{~d}_{A_{0}} v\right\|_{L^{2}(X)}^{2}+\left\|\mathrm{d}_{A_{0}}^{*} v\right\|_{L^{2}(X)}^{2}+\|v\|_{L^{2}(X)}^{2}\right)
$$


Combining of the preceding inequalities gives

$$
\begin{aligned}
\left\|\mathrm{d}_{A} v\right\|_{L^{2}(X)}^{2}+\left\|\mathrm{d}_{A}^{*} v\right\|_{L^{2}(X)}^{2} \geq & \left(\left\|\mathrm{d}_{A_{0}} v\right\|_{L^{2}(X)}^{2}+\left\|\mathrm{d}_{A_{0}}^{*} v\right\|_{L^{2}(X)}^{2}\right)-4 c c_{1}\|a\|_{L^{2 p}(X)}^{2}\|v\|_{L^{2}(X)}^{2} \\
& -4 c_{1} c\|a\|_{L^{2 p}(X)}^{2}\left(\left\|\mathrm{~d}_{A_{0}} v\right\|_{L^{2}(X)}^{2}+\left\|\mathrm{d}_{A_{0}}^{*} v\right\|_{L^{2}(X)}^{2}\right) .
\end{aligned}
$$

Now take $v$ to be an eigenvalue of $\Delta_{A}$ with eigenvalue $\lambda(A)$ and $\|v\|_{L^{2}(X)}=1$ and also suppose that $\left\|A-A_{0}\right\|_{L^{2 p}(X)}$ is small enough that $4 c_{1} c\|a\|_{L^{2 p}(X)}^{2} \leq 1 / 2$. The preceding inequality then gives

$$
\lambda(A) \geq\left(1-4 c_{1} c\|a\|_{L^{2 p}(X)}^{2}\right)\left(\left\|\mathrm{d}_{A_{0}} v\right\|_{L^{2}(X)}^{2}+\left\|\mathrm{d}_{A_{0}}^{*} v\right\|_{L^{2}(X)}^{2}\right)-4 c_{1} c\|a\|_{L^{2 p}(X)}^{2} .
$$

Since $\|v\|_{L^{2}(X)}=1$, we have $\left(\left\|\mathrm{d}_{A_{0}} v\right\|_{L^{2}(X)}^{2}+\left\|\mathrm{d}_{A_{0}}^{*} v\right\|_{L^{2}(X)}^{2}\right) \geq \lambda\left(A_{0}\right)$, hence

$$
\lambda(A) \geq\left(1-4 c_{1} c\|a\|_{L^{2 p}(X)}^{2}\right) \lambda\left(A_{0}\right)-4 c_{1} c\|a\|_{L^{2 p}(X)}^{2} .
$$

To obtain the upper bounded for $\lambda(A)$, we exchange the roles of $A$ and $A_{0}$ that yields the inequality,

$$
\lambda\left(A_{0}\right) \geq\left(1-4 c_{1} c\|a\|_{L^{2 p}(X)}^{2}\right) \lambda(A)-4 c_{1} c\|a\|_{L^{2 p}(X)}^{2} .
$$

\subsection{Uniform positive lower bound for the least eigenvalue of $\Delta_{A}$}

Our results in Section 3.1 assure the continuity of $\lambda(\cdot)$ with respect to the Uhlenbeck topology, and they will be applied here. Before doing this, we recall

Definition 3.5 ([4, Definition 2.4]). Let $G$ be a compact Lie group, $P$ be a $G$-bundle over a closed, smooth manifold $X$ of dimension $n \geq 2$ and endowed with a smooth Riemannian metric $g$. The flat connection, $\Gamma$, is non-degenerate if

$$
\left.\operatorname{ker} \Delta_{\Gamma}\right|_{\Omega^{1}\left(X, \mathfrak{g}_{P}\right)}=\{0\} .
$$

Then we use the results of the continuous of $\lambda[\cdot]$ and compactness of $M(P, g)$ to prove that $\lambda[\cdot]$ has a uniform lower positive bound.

Proposition 3.6. Let $G$ be a compact Lie group, $P$ be a $G$-bundle over a closed, smooth manifold $X$ of dimension $n \geq 2$ and endowed with a smooth Riemannian metric $g$. Then there is a positive constant $\lambda$ with the following significance. Suppose all flat connections on $P$ are non-degenerate. If $\Gamma$ is a flat connection, then

$$
\lambda(\Gamma) \geq \lambda
$$

where $\lambda(\Gamma)$ is as in Definition 3.2 .

Proof. The conclusion is a consequence of the fact that $M(P, g)$ is compact,

$$
\lambda[\cdot]: \quad M(P, g) \ni[\Gamma] \rightarrow \lambda(\Gamma) \in[0, \infty),
$$

to $M(P, g)$ is continuous by Lemma 3.4 , the fact that $\lambda(\Gamma)>0$ for $[\Gamma] \in M(P, g)$.

We consider the open subset of the space $\mathcal{B}(P, g)$ defined by

$$
\mathcal{B}_{\varepsilon}(P, g):=\left\{[A] \in \mathcal{B}(P, g):\left\|F_{A}\right\|_{L^{p}(X)}<\varepsilon\right\},
$$

where $p$ is a constant such that $2 p>n$. Then we have 
Theorem 3.7. Let $G$ be a compact Lie group, $P$ be a $G$-bundle over a closed, smooth manifold $X$ of dimension $n \geq 2$ and endowed with a smooth Riemannian metric $g$, and $2 p>n$. Then there is a positive constant $\varepsilon=\varepsilon(g, n)$ with the following significance. Suppose all flat connections on $P$ are non-degenerate. If $A$ is a smooth connection on $P$ such that

$$
\left\|F_{A}\right\|_{L^{p}(X)} \leq \varepsilon
$$

and $\lambda(A)$ is as in Definition 3.2 , then

$$
\lambda(A) \geq \lambda / 2
$$

where $\lambda$ is the constant in Proposition 3.6.

Proof. For a smooth connection $A$ on $P$ with $\left\|F_{A}\right\|_{L^{p}(X)} \leq \varepsilon$, where $\varepsilon$ is as in the hypotheses of Theorem 2.7. Then there exists a flat connection $\Gamma$ on $P$ and a gauge transformation $g \in L_{2}^{p}(X)$ such that

$$
\left\|g^{*}(A)-\Gamma\right\|_{L_{1, \Gamma}^{p}(X)} \leq C\left\|F_{A}\right\|_{L^{p}(X)}
$$

For $\left\|F_{A}\right\|_{L^{p}(X)}$ sufficiently small, we can apply Lemma 3.4 for $A$ and $\Gamma$ to obtain

$$
\begin{aligned}
\lambda(A) & \geq\left(1-c\left\|g^{*}(A)-\Gamma\right\|_{L^{2 p}(X)}\right) \lambda(\Gamma)-c\left\|g^{*}(A)-\Gamma\right\|_{L^{2 p}(X)} \\
& \geq\left(1-c\left\|g^{*}(A)-\Gamma\right\|_{L_{1, \Gamma}^{p}(X)}\right) \lambda(\Gamma)-c\left\|g^{*}(A)-\Gamma\right\|_{L_{1, \Gamma}^{p}(X)} \\
& \geq \lambda-C c\left\|F_{A}\right\|_{L^{p}(X)}(1+\lambda),
\end{aligned}
$$

here we use Sobolev embedding $L_{1}^{p} \hookrightarrow L^{2 p}$. We choose $\left\|F_{A}\right\|_{L^{p}(X)}$ sufficiently small such that $\mid F_{A} \|_{L^{p}(X)} \leq \frac{\lambda}{2 C c(1+\lambda)}$, then we have $\lambda(A) \geq \lambda / 2$.

\subsection{The case of dimension four}

In this section, we will show a theorem similar to Theorem 3.7 in the case of dimension four, but we only need to suppose that $F_{A}$ with $L^{2}$-norm is sufficiently small. First, we recall a priori the $L^{p}$ estimate for the connection Laplace operator which was proved by Feehan.

Lemma 3.8 ([6, Lemma 35.5]). Let $X$ be a smooth manifold $X$ of dimension $n \geq 4$ and endowed with a smooth Riemannian metric $g$ and $q \in(n, \infty)$. Then there is a positive constant $c=c(g, p)$ with the following significance. Let $r \in\left(\frac{n}{3}, \frac{n}{2}\right)$ be defined by $1 / r=2 / n+1 / q$. Let $A$ is a $C^{\infty}$ connection on a vector bundle $E$ over $X$. If $v \in C^{\infty}(X, E)$, then

$$
\|v\|_{L^{q}(X)} \leq c\left(\left\|\nabla_{A}^{*} \nabla_{A} v\right\|_{L^{r}(X)}+\|v\|_{L^{r}(X)}\right) .
$$

We now apply Lemma 3.8 to $E=\Omega^{1} \otimes \mathfrak{g}_{P}$, then we give a priori $L^{p}$-estimate for $\Delta_{A}$.

Lemma 3.9. Let $G$ be a compact Lie group, $P$ be a $G$-bundle over a closed, smooth manifold $X$ of dimension $n \geq 4$ and endowed with a smooth Riemannian metric $g$ and $q \in(n, \infty)$. Then there are positive constants, $c=c(g)$ and $\varepsilon=\varepsilon(g)$, with the following significance. Let $r \in\left(\frac{n}{3}, \frac{n}{2}\right)$ defined by $1 / r=2 / n+1 / q$. Let $A$ be a $C^{\infty}$ connection on $P$ that each obeys the curvature bounded (3.2). If $v \in \Omega^{1}\left(X, \mathfrak{g}_{P}\right)$, then

$$
\|v\|_{L^{q}(X)} \leq c\left(\left\|\Delta_{A} v\right\|_{L^{r}(X)}+\|v\|_{L^{r}(X)}\right) .
$$


Proof. For $v \in \Omega^{1}\left(X, \mathfrak{g}_{P}\right)$, from the Weitzenböck formula, we have

$$
\Delta_{A} v=\nabla_{A}^{*} \nabla_{A} v+\operatorname{Ric} \cdot v+\left\{v, F_{A}\right\}
$$

Hence

$$
\left\|\Delta_{A} v\right\|_{L^{r}(X)} \leq\left\|\nabla_{A}^{*} \nabla_{A} v\right\|_{L^{r}(X)}+c\|v\|_{L^{r}(X)}+\left\|\left\{v, F_{A}\right\}\right\|_{L^{r}(X)},
$$

for some $c=c(g)$. Since $1 / r=2 / n+1 / q$ by hypothesis, we see that

$$
\left\|\left\{v, F_{A}\right\}\right\|_{L^{r}(X)} \leq c\left\|F_{A}\right\|_{L^{n / 2}(X)}\|v\|_{L^{q}(X)},
$$

for some $c=c(g)$. Combining the preceding inequalities with the equation (3.4) we get

$$
\|v\|_{L^{q}(X)} \leq\left\|\nabla_{A}^{*} \nabla_{A} v\right\|_{L^{r}(X)}+c\|v\|_{L^{r}(X)}+c\left\|F_{A}\right\|_{L^{n / 2}(X)}\|v\|_{L^{q}(X)},
$$

for some $c=c(g, q)$. Provided $c\left\|F_{A}\right\|_{L^{n / 2}(X)} \leq 1 / 2$, the rearrangement gives (3.4).

Hence, following the idea of [8, Lemma 35.13], we also have a useful lemma.

Lemma 3.10 ( $L^{p}$ continuity of least eigenvalue of $\Delta_{A}$ with respect to the connection for $2<p \leq 4)$. Let $G$ be a compact Lie group, $P$ be a $G$-bundle over a closed, smooth four-manifold $X$ and endowed with a smooth Riemannian metric $g$. Then there are positive constants, $c=c(g)$ and $\varepsilon=\varepsilon(g)$, with the following significance. If $A_{0}$ and $A$ are $C^{\infty}$ connections on $P$ that each obeys the curvature bounded (3.2), we denote $a:=A-A_{0}$, then $\lambda(A)$ satisfies

$$
\lambda(A) \geq \lambda\left(A_{0}\right)-c_{0}\left(1+\lambda^{2}(A)\right)\|a\|_{L^{p}(X)}^{2},
$$

and

$$
\lambda(A) \leq \lambda\left(A_{0}\right)+c_{0}\left(1+\lambda^{2}\left(A_{0}\right)\right)\|a\|_{L^{p}(X)}^{2} .
$$

Proof. For convenience, write $a:=A-A_{0} \in L^{p}\left(X, \Omega^{1} \otimes \mathfrak{g}_{P}\right)$. Define $q \in[2, \infty)$ by $1 / 2=$ $1 / p+1 / q$ and consider $v \in L_{1}^{2}\left(X, \Omega^{1} \otimes \mathfrak{g}_{P}\right)$. We use $\mathrm{d}_{A} v=\mathrm{d}_{A_{0}} v+[a, v]$ and the Höler inequalities to give

$$
\begin{aligned}
\left\|\mathrm{d}_{A} v\right\|_{L^{2}(X)}^{2} & =\left\|\mathrm{d}_{A_{0}} v+[a, v]\right\|_{L^{2}(X)}^{2} \geq\left\|\mathrm{d}_{A_{0}} v\right\|_{L^{2}(X)}^{2}-\|[a, v]\|_{L^{2}(X)}^{2} \\
& \geq\left\|\mathrm{d}_{A_{0}} v\right\|_{L^{2}(X)}^{2}-2\|a\|_{L^{p}(X)}^{2}\|v\|_{L^{q}(X)}^{2} .
\end{aligned}
$$

Similarly, $\mathrm{d}_{A}^{*} v=\mathrm{d}_{A_{0}}^{*} v \pm *[a, * v]$ and

$$
\begin{aligned}
\left\|\mathrm{d}_{A}^{*} v\right\|_{L^{2}(X)}^{2} & =\left\|\mathrm{d}_{A_{0}}^{*} v \pm *[a, * v]\right\|_{L^{2}(X)}^{2} \geq\left\|\mathrm{d}_{A_{0}}^{*} v\right\|_{L^{2}(X)}^{2}-\|*[a, * v]\|_{L^{2}(X)}^{2} \\
& \geq\left\|\mathrm{d}_{A_{0}}^{*} v\right\|_{L^{2}(X)}^{2}-2\|a\|_{L^{p}(X)}^{2}\|v\|_{L^{q}(X)}^{2}
\end{aligned}
$$

For $p>4$, we have $2 \leq q<4$ and $\|v\|_{L^{q}(X)} \leq(\operatorname{vol}(X))^{1 / q-1 / 4}\|v\|_{L^{4}(X)}$, while for $2<p \leq 4$, we have $4 \leq q<\infty$. Therefore, it suffices to consider the case $4 \leq q<\infty$. Applying the a priori estimate (3.4) and $r \in(4 / 3,2)$ defined by $1 / r=1 / 2+1 / q$, we get

$$
\|v\|_{L^{q}(X)}^{2} \leq c\left(\left\|\Delta_{A} v\right\|_{L^{4 / 3}(X)}^{2}+\|v\|_{L^{r}(X)}^{2}\right) \leq c\left(\left\|\Delta_{A} v\right\|_{L^{2}(X)}^{2}+\|v\|_{L^{r}(X)}^{2}\right) .
$$

Combining the preceding inequalities, we get

$$
\begin{aligned}
& \left\|\mathrm{d}_{A} v\right\|_{L^{2}(X)}^{2}+\left\|\mathrm{d}_{A}^{*} v\right\|_{L^{2}(X)}^{2} \\
& \quad \geq\left\|\mathrm{d}_{A_{0}} v\right\|_{L^{2}(X)}^{2}+\left\|\mathrm{d}_{A_{0}}^{*} v\right\|_{L^{2}(X)}^{2}-2 c_{1}\|a\|_{L^{p}(X)}^{2}\left(\left\|\Delta_{A} v\right\|_{L^{r}(X)}^{2}+\|v\|_{L^{r}(X)}^{2}\right) \\
& \quad \geq\left\|\mathrm{d}_{A_{0}} v\right\|_{L^{2}(X)}^{2}+\left\|\mathrm{d}_{A_{0}}^{*} v\right\|_{L^{2}(X)}^{2}-2 c_{0}\|a\|_{L^{p}(X)}^{2}\left(\left\|\Delta_{A} v\right\|_{L^{2}(X)}+\|v\|_{L^{2}(X)}^{2}\right),
\end{aligned}
$$


for $c_{0}=c_{0}(p, q)=2 c_{1} \operatorname{vol}(X)^{2 / q}$, using the fact that $\|v\|_{L^{r}(X)} \leq \operatorname{vol}(X)^{1 / q}\|v\|_{L^{2}(X)}$ for $r \in$ $(4 / 3,2)$ and $1 / r=1 / 2+1 / q$. By taking $v \in L_{1}^{2}\left(X, \Omega^{1} \otimes \mathfrak{g}_{P}\right)$ to be an eigenvector of $\Delta_{A}$ with eigenvalue $\lambda(A)$ such that $\|v\|_{L^{2}(X)}=1$ and noting that $\left\|\Delta_{A} v\right\|_{L^{2}(X)}=\lambda(A)$ we obtain the bound

$$
\lambda(A) \geq\left\|\mathrm{d}_{A_{0}} v\right\|_{L^{2}(X)}^{2}+\left\|\mathrm{d}_{A_{0}}^{*} v\right\|_{L^{2}(X)}^{2}-c_{0}\left(1+\lambda^{2}(A)\right)\|a\|_{L^{p}(X)}^{2} .
$$

But $\left\|\mathrm{d}_{A_{0}} v\right\|_{L^{2}(X)}^{2}+\left\|\mathrm{d}_{A_{0}}^{*} v\right\|_{L^{2}(X)}^{2} \geq \lambda\left(A_{0}\right)$ and thus we have the inequality

$$
\lambda(A) \geq \lambda\left(A_{0}\right)-c_{0}\left(1+\lambda^{2}(A)\right)\|a\|_{L^{p}(X)}^{2} .
$$

Interchanging of the roles $A$ and $A_{0}$ in the preceding derivation yields

$$
\lambda(A) \leq \lambda\left(A_{0}\right)+c_{0}\left(1+\lambda^{2}\left(A_{0}\right)\right)\|a\|_{L^{p}(X)}^{2} .
$$

We consider a sequence of $C^{\infty}$ connections $\left\{A_{i}\right\}_{i \in \mathbb{N}}$ on $P$ such that $\sup \left\|F_{A_{i}}\right\|_{L^{2}(X)}<\infty$. We denote

$$
\Sigma=\left\{x \in X: \lim _{r \searrow 0} \limsup _{i \rightarrow \infty}\left\|F_{A_{i}}\right\|_{L^{2}\left(B_{r}\right)(x)}^{2} \geq \tilde{\varepsilon}\right\},
$$

the constant $\tilde{\varepsilon} \in(0,1]$ as in $\left[15\right.$, Theorem 3.2]. We can see $\Sigma$ is a finite points $\left\{x_{1}, \ldots, x_{L}\right\}$ in $X$. In our article, we consider the open subset of the space $\mathcal{B}(P, g)$ defined by

$$
\mathcal{B}_{\varepsilon}=\left\{[A] \in \mathfrak{B}(P, g):\left\|F_{A}\right\|_{L^{2}(X)}<\varepsilon\right\} .
$$

We can choose $\varepsilon$ sufficiently small such that any sequence $\left\{A_{i}\right\}_{i \in \mathbb{N}}$ has the empty set $\Sigma$. From [15, Theorem 3.1] and [6, Theorem 35.15], we have

Theorem 3.11. Let $G$ be a compact Lie group and $P$ be a principal $G$-bundle over a close, smooth four-dimensional $X$ with Riemannian metric $g$. If $\left\{A_{i}\right\}_{i \in \mathbb{N}}$ is a sequence of $C^{\infty}$ connections on $P$ such that $\left\|F_{A_{i}}\right\|_{L^{2}(X)} \leq \varepsilon$, there exists a subsequence, a countable set of arbitrarily small geodesic balls $\left\{B_{\alpha}\right\}_{\alpha \in \mathbb{N}}$ covering $X, C^{\infty}$-sections

$$
\sigma_{\alpha, i}: \quad B_{\alpha} \rightarrow P, \quad A_{\alpha} \in L_{1}^{2}\left(B_{\alpha} ; \Omega^{1} B_{\alpha} \otimes \mathfrak{g}\right), \quad g_{\alpha \beta} \in L_{1}^{4}\left(B_{\alpha} \cap B_{\beta} ; G\right),
$$

such that

(1) $\mathrm{d}^{*} A_{\alpha}(i)=0$, for all $i$ sufficiently large,

(2) $\mathrm{d}^{*} A_{\alpha}=0$,

(3) $g_{\alpha \beta}(i) \rightarrow g_{\alpha \beta}$ weakly in $L_{1}^{4}\left(B_{\alpha} \cap B_{\beta} ; G\right)$,

(4) $F_{\alpha}(i) \rightarrow F_{\alpha}$ weakly in $L^{2}\left(B_{\alpha} ; \Omega^{2} B_{\alpha} \otimes \mathfrak{g}\right)$,

(5) the sequence $\left\{A_{\alpha}(i)\right\}_{i \in \mathbb{N}}$ obeys

(a) $A_{\alpha}(i) \rightarrow A_{\alpha}$ weakly in $L_{1}^{2}\left(B_{\alpha} ; \Omega^{1} B_{\alpha} \otimes \mathfrak{g}\right)$, and

(b) $A_{\alpha}(i) \rightarrow A_{\alpha}$ strongly in $L^{p}\left(B_{\alpha} ; \Omega^{1} B_{\alpha} \otimes \mathfrak{g}\right)$ for $1 \leq p<4$,

(6) $A_{\alpha}=g_{\alpha \beta}^{-1} A_{\beta} g_{\alpha \beta}+g_{\alpha \beta}^{-1} d g_{\alpha \beta}$.

Here $A_{\alpha}(i)=\sigma_{\alpha}^{*} A_{i}, F_{\alpha}=\mathrm{d} A_{\alpha}+\left[A_{\alpha}, A_{\alpha}\right], F_{\alpha}(i)=\mathrm{d} A_{\alpha}(i)+\left[A_{\alpha}(i), A_{\alpha}(i)\right]$ and $\mathrm{d}^{*}$ is the formal adjoint of $\mathrm{d}$ with respect to the flat metric defined by a choice of geodesic normal coordinates on $B_{\alpha}$.

From [15, Theorem 4.3] and [6, Theorem 35.17], we have 
Theorem 3.12. Let $G$ be a compact Lie group and $P$ be a principal $G$-bundle over a close, smooth four-dimensional $X$ with Riemannian metric $g$. If $\left\{A_{i}\right\}_{i \in \mathbb{N}}$ is a sequence of $C^{\infty}$ connections on $P$, in the sense that

$$
\mathrm{YM}\left(A_{i}\right) \searrow 0 \quad \text { as } i \rightarrow \infty
$$

then the following hold, for each $\alpha, \beta \in \mathbb{N}$,

(1) $A_{\alpha} \in C^{\infty}\left(B_{\alpha} ; \Omega^{1} B_{\alpha} \otimes \mathfrak{g}\right)$ and a solution to the flat connection,

(2) $g_{\alpha \beta} \in C^{\infty}\left(B_{\alpha} \bigcap B_{\beta} ; G\right)$,

(3) the sequence, $\left\{A_{\alpha}\right\}_{\alpha \in \mathbb{N}}$ and $\left\{g_{\alpha \beta}\right\}_{\alpha, \beta \in \mathbb{N}}$ define a $C^{\infty}$ flat connection $A_{\infty}$ on a principal $G$-bundle $P_{\infty}$ over $X$.

Then, we have the useful

Corollary 3.13. Assume the hypotheses of Theorem 3.12, then

$$
\lim _{i \rightarrow \infty} \lambda\left(A_{i}\right)=\lambda\left(A_{\infty}\right)
$$

where $\lambda(\Gamma)$ is as in Definition 3.2 .

Proof. From Theorem 3.12, $\sigma_{\alpha, i}^{*} A_{i} \rightarrow \sigma^{*} A_{\infty}$ weakly in $L_{1}^{2}\left(B_{\alpha}, \Omega^{1} B_{\alpha} \otimes \mathfrak{g}_{P}\right)$. For $L_{1}^{2} \Subset L^{p}$, $(2<p<4)$, hence

$$
\left\|\sigma_{\alpha, i}^{*} A_{i}-\sigma^{*} A_{\infty}\right\|_{L^{p}\left(B_{\alpha}\right)} \rightarrow 0 \quad \text { as } \quad i \rightarrow \infty,
$$

for sequences of local sections $\left\{\sigma_{\alpha, m}\right\}_{m \in \mathbb{N}}$ of $P \uparrow B_{\alpha}$ and a local section $\sigma_{\alpha}$ of $P_{\infty} \uparrow B_{\alpha}$ and

$$
\left\|A_{i}-A_{\infty}\right\|_{L^{p}(X)} \leq \sum_{a}\left\|\sigma_{\alpha, m}^{*} A_{m}-\sigma^{*} A_{\infty}\right\|_{L^{p}\left(B_{\alpha}\right)}
$$

Hence from Lemma 3.10, we have

$$
\lim _{i \rightarrow \infty} \lambda\left(A_{i}\right) \geq \lambda\left(A_{\infty}\right)-c_{0}\left(1+\lim _{i \rightarrow \infty} \lambda^{2}\left(A_{i}\right)\right) \lim _{i \rightarrow \infty}\left\|a_{i}\right\|_{L^{p}(X)}
$$

and

$$
\lim _{i \rightarrow \infty} \lambda\left(A_{i}\right) \leq \lambda\left(A_{\infty}\right)+c_{0}\left(1+\lambda^{2}\left(A_{\infty}\right)\right) \lim _{i \rightarrow \infty}\left\|a_{i}\right\|_{L^{p}(X)} .
$$

Then we obtain

$$
\lim _{i \rightarrow \infty} \lambda\left(A_{i}\right)=\lambda\left(A_{\infty}\right) .
$$

Then we have

Theorem 3.14. Let $G$ be a compact Lie group, $P$ be a G-bundle over a closed, smooth 4manifold and endowed with a smooth Riemannian metric $g$. Then there is a positive constant $\varepsilon=\varepsilon(g) \in(0,1]$ with the following significance. Suppose all flat connections on $P$ are nondegenerate. If $A$ is a $C^{\infty}$ connection on $P$ such that

$$
\left\|F_{A}\right\|_{L^{2}(X)} \leq \varepsilon
$$

and $\lambda(A)$ is as in Definition 3.2 , then

$$
\lambda(A) \geq \frac{\lambda}{2}
$$

where $\lambda$ is the positive constant in Proposition 3.6.

Proof. Suppose that the constant $\lambda \in(0,1]$ does not exist. We may then choose a sequence $\left\{A_{i}\right\}_{i \in \mathbb{N}}$ of connections on $P$ such that $\left\|F_{A_{i}}\right\|_{L^{2}(X)} \rightarrow 0$ and $\lambda\left(A_{i}\right) \rightarrow 0$ as $i \rightarrow \infty$. Since $\lim _{i \rightarrow \infty} \lambda\left(A_{i}\right)=\lambda\left(A_{\infty}\right)$ and $\lambda\left(A_{\infty}\right)>0$ by $A_{\infty}$ is a flat connection, then it contradicts our initial assumption regarding the sequence $\left\{A_{i}\right\}_{i \in \mathbb{N}}$. 


\section{Proof of the main Theorem 1.2}

Now, we begin to prove the energy gap result for the complex Yang-Mills equations. At first, we prove the complex Yang-Mills equations will be reduce to pure Yang-Mills equation under the certain conditions for $g, G, P$, and $X$.

Proposition 4.1. Let $X$ be a closed, oriented, smooth Riemannian manifold of dimension $n \geq 2$ with smooth Riemannian metric $g, P$ be a $G$-bundle over $X$, let $2 p>n$ when $n \neq 4$ or $p \geq 2$ when $n=4$. Then there exists a positive constant $\varepsilon=\varepsilon(g, n, p)$ with the following significance. Suppose that all flat connections on $P$ are non-degenerate. If the pair $(A, \phi)$ is a $C^{\infty}$-solution of complex Yang-Mills equations over $X$ and the curvature $F_{A}$ of connection $A$ obeys

$$
\left\|F_{A}\right\|_{L^{p}(X)} \leq \varepsilon
$$

then $\phi$ vanishes.

Proof. If we suppose that

$$
\left\|F_{A}\right\|_{L^{p}(X)} \leq \varepsilon
$$

where $p$ and $\varepsilon$ are the constants satisfying the hypotheses in Theorems 3.7 and 3.14, then there exists a positive constant $\lambda$ such that

$$
\left\|\mathrm{d}_{A} v\right\|_{L^{2}(X)}^{2}+\left\|\mathrm{d}_{A}^{*} v\right\|_{L^{2}(X)}^{2} \geq \lambda / 2\|v\|_{L^{2}(X)}^{2}, \quad \forall v \in \Omega^{1}\left(X, \mathfrak{g}_{P}\right) .
$$

We have an identity for the solution of complex Yang-Mills equations

$$
\left\|\mathrm{d}_{A} \phi\right\|_{L^{2}(X)}^{2}+\left\|\mathrm{d}_{A}^{*} \phi\right\|_{L^{2}(X)}^{2}+2\|\phi \wedge \phi\|_{L^{2}(X)}^{2}-2\left\langle F_{A}, \phi \wedge \phi\right\rangle_{L^{2}(X)}=0 .
$$

Hence, we have

$$
\begin{aligned}
\lambda / 2\|\phi\|_{L^{2}(X)}^{2} & \leq\left\|\mathrm{d}_{A} \phi\right\|_{L^{2}(X)}^{2}+\left\|\mathrm{d}_{A}^{*} \phi\right\|_{L^{2}(X)}^{2} \leq\left|\left\langle F_{A}, \phi \wedge \phi\right\rangle_{L^{2}(X)}\right| \\
& \leq C\left\|F_{A}\right\|_{L^{p}(X)}\|\phi\|_{L^{2 q}(X)}^{2} \leq C\left\|F_{A}\right\|_{L^{p}(X)}\|\phi\|_{L^{2}(X)}^{2}
\end{aligned}
$$

where $1 / q=1-1 / p, C=C(g, p)$. We can choose $\left\|F_{A}\right\|_{L^{p}(X)} \leq \varepsilon$ sufficiently small such that $C \varepsilon \leq \lambda / 4$, then the extra field $\phi$ vanishes.

Proof Theorem 1.2. From Proposition 4.1, the complex Yang-Mills equations reduce to the pure Yang-Mills equation $\mathrm{d}_{A}^{*} F_{A}=0$ and the curvature obeys $\left\|F_{A}\right\|_{L^{p}(X)} \leq \varepsilon$, then by the energy gap of Yang-Mills connection, we obtain that the connection $A$ is flat.

Proof Corollary 1.4. For a smooth solution $(A, \phi)$ of complex flat connection, from the identity $F_{A}=\phi \wedge \phi$ and we apply Theorem 2.5 to obtain

$$
\left\|F_{A}\right\|_{L^{p}(X)} \leq\|\phi \wedge \phi\|_{L^{p}(X)} \leq C\|\phi\|_{L^{2}(X)}^{2},
$$

where $C=C(g, n, p)$. We can choose $\|\phi\|_{L^{2}(X)}$ sufficiently small such that $\left\|F_{A}\right\|_{L^{p}(X)} \leq \varepsilon$, where $\varepsilon$ is the constant in Theorem 1.2. Then we can prove that $\phi$ vanishes and $A$ is a flat connection.

It is easy to see the map $(A, \phi) \mapsto\|\phi\|_{L^{2}(X)}$ is continuous, then the moduli space of complex flat connections is non-connected. 


\section{Acknowledgements}

I would like to thank Karen Uhlenbeck and Michael Gagliardo for helpful comments regarding their article [9] and Paul Feehan for helpful comments regarding his articles [6, 7, 8]. I thank the anonymous referees for a careful reading of my article and helpful comments and corrections. This work is partially supported by Wu Wen-Tsun Key Laboratory of Mathematics of Chinese Academy of Sciences at USTC.

\section{References}

[1] Bourguignon J.-P., Lawson H.B., Stability and isolation phenomena for Yang-Mills fields, Comm. Math. Phys. 79 (1981), 189-230.

[2] Bourguignon J.-P., Lawson H.B., Simons J., Stability and gap phenomena for Yang-Mills fields, Proc. Nat. Acad. Sci. USA 76 (1979), 1550-1553.

[3] Dodziuk J., Min-Oo M., An $L_{2}$-isolation theorem for Yang-Mills fields over complete manifolds, Compositio Math. 47 (1982), 165-169.

[4] Donaldson S.K., Floer homology groups in Yang-Mills theory, Cambridge Tracts in Mathematics, Vol. 47, Cambridge University Press, Cambridge, 2002.

[5] Donaldson S.K., Kronheimer P.B., The geometry of four-manifolds, Oxford Mathematical Monographs, Oxford Science Publications, The Clarendon Press, Oxford University Press, New York, 1990.

[6] Feehan P.M.N., Global existence and convergence of smooth solutions to Yang-Mills gradient flow over compact four-manifolds, arXiv:1409.1525.

[7] Feehan P.M.N., Energy gap for Yang-Mills connections, I: Four-dimensional closed Riemannian manifolds, Adv. Math. 296 (2016), 55-84, arXiv:1412.4114.

[8] Feehan P.M.N., Energy gap for Yang-Mills connections, II: Arbitrary closed Riemannian manifolds, Adv. Math. 312 (2017), 547-587, arXiv:1502.00668.

[9] Gagliardo M., Uhlenbeck K., Geometric aspects of the Kapustin-Witten equations, J. Fixed Point Theory Appl. 11 (2012), 185-198, arXiv:1401.7366.

[10] Gerhardt C., An energy gap for Yang-Mills connections, Comm. Math. Phys. 298 (2010), 515-522, arXiv:0908.0767.

[11] Gilbarg D., Trudinger N.S., Elliptic partial differential equations of second order, Grundlehren der Mathematischen Wissenschaften, Vol. 224, 2nd ed., Springer-Verlag, Berlin, 1983.

[12] Hitchin N.J., The self-duality equations on a Riemann surface, Proc. London Math. Soc. 55 (1987), $59-126$.

[13] Huang T., A proof on energy gap for Yang-Mills connection, arXiv:1704.02772.

[14] Min-Oo M., An $L_{2}$-isolation theorem for Yang-Mills fields, Compositio Math. 47 (1982), $153-163$.

[15] Sedlacek S., A direct method for minimizing the Yang-Mills functional over 4-manifolds, Comm. Math. Phys. 86 (1982), 515-527.

[16] Simpson C.T., Constructing variations of Hodge structure using Yang-Mills theory and applications to uniformization, J. Amer. Math. Soc. 1 (1988), 867-918.

[17] Taubes C.H., Self-dual Yang-Mills connections on non-self-dual 4-manifolds, J. Differential Geom. 17 (1982), 139-170.

[18] Uhlenbeck K.K., Removable singularities in Yang-Mills fields, Comm. Math. Phys. 83 (1982), 11-29.

[19] Uhlenbeck K.K., Connections with $L^{p}$ bounds on curvature, Comm. Math. Phys. 83 (1982), 31-42.

[20] Uhlenbeck K.K., The Chern classes of Sobolev connections, Comm. Math. Phys. 101 (1985), 449-457.

[21] Wehrheim K., Uhlenbeck compactness, EMS Series of Lectures in Mathematics, European Mathematical Society (EMS), Zürich, 2004. 